\title{
New perspective in statistical modeling of wall-bounded turbulence
}

\author{
Zhen-Su She · Xi Chen · You Wu • Fazle Hussain
}

Received: 24 August 2010 / Revised: 29 September 2010 / Accepted: 27 October 2010 / Published online: 3 December 2010

(C) The Author(s) 2010. This article is published with open access at Springerlink.com

\begin{abstract}
Despite dedicated effort for many decades, statistical description of highly technologically important wall turbulence remains a great challenge. Current models are unfortunately incomplete, or empirical, or qualitative. After a review of the existing theories of wall turbulence, we present a new framework, called the structure ensemble dynamics (SED), which aims at integrating the turbulence dynamics into a quantitative description of the mean flow. The SED theory naturally evolves from a statistical physics understanding of non-equilibrium open systems, such as fluid turbulence, for which mean quantities are intimately coupled with the fluctuation dynamics. Starting from the ensemble-averaged Navier-Stokes (EANS) equations, the theory postulates the existence of a finite number of statistical states yielding a multi-layer picture for wall turbulence. Then, it uses order functions (ratios of terms in the mean momentum as well as energy equations) to characterize the states and transitions between states. Application of the SED analysis to an incompressible channel flow and a compressible turbulent boundary layer shows that the order functions successfully reveal the multi-layer structure for wall-bounded turbulence, which arises as a quantitative extension of the traditional view in terms of sub-layer, buffer layer, log layer and wake.
\end{abstract}

The project was supported by the National Natural Science Foundation of China (90716008) and the National Basic Research Program of China (2009CB724100).

\section{Z.-S. She $(\bowtie) \cdot X$. Chen $\cdot$ Y. Wu}

State Key Laboratory for Turbulence and Complex Systems and Department of Mechanical and Aerospace Engineering,

College of Engineering, Peking University, 100871 Beijing, China

e-mail: she@pku.edu.cn

F. Hussain

Department of Mechanical Engineering, University of Houston, Houston, TX 77204-4792, USA
Furthermore, an idea of using a set of hyperbolic functions for modeling transitions between layers is proposed for a quantitative model of order functions across the entire flow domain. We conclude that the SED provides a theoretical framework for expressing the yet-unknown effects of fluctuation structures on the mean quantities, and offers new methods to analyze experimental and simulation data. Combined with asymptotic analysis, it also offers a way to evaluate convergence of simulations. The SED approach successfully describes the dynamics at both momentum and energy levels, in contrast with all prevalent approaches describing the mean velocity profile only. Moreover, the SED theoretical framework is general, independent of the flow system to study, while the actual functional form of the order functions may vary from flow to flow. We assert that as the knowledge of order functions is accumulated and as more flows are analyzed, new principles (such as hierarchy, symmetry, group invariance, etc.) governing the role of turbulent structures in the mean flow properties will be clarified and a viable theory of turbulence might emerge.

Keywords Wall turbulence - Statistical modeling · Structure ensemble dynamics · Order function · Multi-layer

\section{Introduction}

Turbulence is an intensely researched, engaging problem for over a century, since the time of Osborne Reynolds. However, rules for identifying turbulent flow structures and ways to predict mean quantities remain elusive. Wall-bounded turbulent flow has attracted considerable recent attention because of its relative simplicity and its direct relevance to engineering applications, and its study has engendered notable physical understanding of turbulence phenomena. Despite 
great experimental and computational efforts, theoretical description of wall turbulence remains greatly wanting. Mean velocity and Reynolds stress profiles are mostly empirical; asymptotic laws such as log-law or power-law address, at most, only limited portions of the flow domain, with lively controversies; discussions of mechanisms of turbulence production and vortex generation are remote to quantitative modeling of mean quantities, and so on. More importantly, theoretical studies lack a coherent setting to guide experiment or computation.

Theory devoted to predicting mean properties of flows is usually called the closure. Three kinds of closure theories have been developed over the past several decades. The earliest one advanced by Kolmogorov [1] in 1940s expresses unknown correlation terms in the ensemble averaged Navier-Stokes (EANS) equations in terms of correlation variables (which are being solved) using some "ad hoc" closure assumptions commonly referred to as Reynolds averaged Navier-Stokes (RANS) modeling. Solutions of RANS equation, usually obtained numerically, compute the mean velocity profile. This approach is the core of industrial computational fluid dynamics (CFD) software. In aerospace industry, RANS-based CFD tools are widely used, but empirical constants need to be adjusted using large sets of experimental database. Drag coefficient prediction using CFD software can reach an accuracy of $10^{-3}$ to $10^{-4}$, with the error larger than experimental data by one order of magnitude. Further improvement seems to require a fundamental change in the artificial nature of RANS formulation.

Current RANS models for wall turbulence are essentially based on Prandtl's boundary layer theory. For flows passing by a nearly flat plate, RANS models are acceptable because Prandtl's theory is qualitatively correct. However, for flow passing over a complex object such as a wing or flap, turbulent mean flow structures are very little known theoretically. Consequently, there exists no reliable basis for guaranteeing accurate CFD computations of flows around a whole airplane. A theory of mean flow for complex geometries is needed for making a decisive progress, and for that, a quantitative turbulent boundary layer theory is a pre-requisite.

How far are we away from such a quantitative theory of turbulent boundary layer? During the past several decades, experiments, direct numerical simulations (DNS) and largeeddy simulations (LES) have provided a huge database about detailed flow structures both in canonical settings such as channel, pipe and boundary layer, and in more complex environments [2-4]. The fact that a quantitative theory is still out of sight, despite of tons of DNS and LES data, is proof of missing good concepts or a good framework. A productive framework would enable us to extract relevant information from DNS or LES data and to characterize quantitative effects of turbulent structures on the means. Once such a framework is in place, DNS or LES studies would be better guided and quantitative closure descriptions would be uncovered.

In this paper, we discuss a new perspective for such a theoretical framework from a statistical physics and complex system point of view. A key claim of the new approach is that turbulent flows need to be treated as an "open" problem, and the effects of fluctuations on the mean should not be described in a fixed functional form, because a variety of flow structures in different environments can generate different eddy effects, corresponding to different functional forms. In other words, a viable theoretical framework capable of describing complex flows ought to be general regarding closure schema. Specific closure schema for specific flow can be inspired from DNS results. A framework needs to provide concepts which enable the extraction of such an appropriate closure schema for each flow from empirical (DNS or LES) data, which is unfortunately missing. The new approach proposed here fulfills this need. In the long run, as more flows are studied under the new framework, universal principles that govern some aspects of the mean quantities will emerge.

The paper is organized as follows. In Sect. 2, we give a review of relevant closure theories. The statistical physics background and main ideas of the new approach are explained in Sect. 3. In Sect. 4, we present a detailed framework for the study of wall-bounded turbulent flows, including universal balance principles, order functions and quantitative models, with several key results of the new approach obtained for incompressible turbulent channel flows and a compressible turbulent boundary layer. Section 5 contains a discussion of several applications of the new approach, including a new second-order closure schema, a guide to DNS study of wall turbulence in general and a schema to evaluate simulation data. At the end, the future of turbulence theory is discussed.

\section{A review of the relevant closure theories}

Theoretical study in wall-bounded turbulence mostly focuses on the prediction of (time-averaged) mean velocity, and other related mean properties determining drag, lift, heat transfer and other quantities of engineering interest. Here we review the relevant closure theories, divided into two kinds as follows.

\subsection{Scaling analysis}

The first important issue in determining mean velocity is the choice of right scaling for the velocity at large Reynolds number $(R e)$. According to Prandtl [5], the law of wall is

$$
\frac{U}{u_{\tau}}=f\left(y^{+}\right)
$$


where $u_{\tau}$ is the friction velocity. von Karman [6] and Clauser [7] proposed the so-called velocity defect law,

$\frac{U_{e}-U}{u_{\tau}}=g(\eta)$,

where $U_{e}$ is the free stream velocity in the outer flow, $\eta \equiv y / \Delta$ and $\Delta$ is a characteristic thickness of the boundary layer.

However, there are different opinions about the scaling of the outer flow. George and Castillo [8] argued that only $U_{e}$ is the right velocity scaling for the outer flow, which is consistent with the similarity solution for the Prandtl's boundary layer equation at infinite $R e$. The criterion is based on an asymptotic invariant principle (AIP) that all of the coefficients in the rescaled momentum equation have the same dependence on $x$. However, Jones et al. [9] proved that this criterion can not eliminate $u_{\tau}$ scaling, and the wake function, for example, can be one of the similarity solutions. Nevertheless, their claims can not be checked because of the infinite $R e$ needed. Another outer scaling is the Zagarola and Smits [10] (hereafter referred to as ZS) scaling, by which the velocity defect shows a better similarity. Until now, the debate on the validity of von Karman scaling, George scaling and ZS scaling is alive. Note that all of these scalings can fit the friction data quite well by adjusting coefficients [11]. Therefore, a relevant issue is: Which of the scalings is valid for a wider range of $R e$ ?

Next step is to determine matching conditions. From Eqs. (1) and (2), Millikan [12] obtained a matching equation

$y^{+} \frac{\mathrm{d} f\left(y^{+}\right)}{\mathrm{d} y^{+}}=\eta \frac{\mathrm{d} g(\eta)}{\mathrm{d} \eta}$.

If each side of this formula is assumed to be constant, a log-law profile follows. This scale separation assumption between $y^{+}$and $\eta$ is reasonable when $R e \rightarrow \infty$. Millikan also pointed out that if the velocity defect form Eq. (2) is taken to be $U / U_{e}=g(\eta)$, then

$\frac{y^{+}}{f\left(y^{+}\right)} \frac{\mathrm{d} f\left(y^{+}\right)}{\mathrm{d} y^{+}}=\frac{\eta}{g(\eta)} \frac{\mathrm{d} g(\eta)}{\mathrm{d} \eta}$,

which yields a power law.

Since then, models have been developed in two directions: log-law and power-law. Nickels [13] and Monkewitz et al. [14] (hereafter referred to as MCN) are examples of the first kind (see next subsection), while George et al. suggested a power law for TBL [8] and a log low for channel [15] (hereafter referred to as WCG). Furthermore, at a finite $R e$, setting $R e$ - or $y$-dependent constants in Eq. 3 gives rise to different asymptotic formulas.

For channel flow, the WCG theory assumes that a solution for the overlap region is given by

$\frac{U}{u_{*}}=\frac{1}{\kappa(R e)} \ln \left[y^{+}+a^{+}(R e)\right]+B_{i}(R e)$.
Then, they claimed the existence of the asymptotic log-law for channel and pipe flows, with $R e$-dependent coefficients $\kappa$ and $a^{+}$; the latter is speculated to be related to a so-called meso-layer $\left(30 \lesssim y^{+} \lesssim 300\right)$.

Buschmann and Gad-el-hak [16] suggested that a more complex functional form is needed to describe the mean velocity, and proposed a generalized log-law to solve Eq. 3 at a finite $R e$. By introducing expansions

$f=\sum_{i} f_{i} / R e_{\tau}^{i}, \quad g=\sum_{i} g_{i} / R e_{\tau}^{i}$,

where $i=0,1,2, \ldots$, they obtain a zeroth order equation as

$$
\begin{aligned}
& \frac{\mathrm{d} f_{0}}{\mathrm{~d} \log y^{+}}=c_{1}+E_{1} / y^{+}, \\
& \frac{\mathrm{d} g_{0}}{\mathrm{~d} \log \eta}=c_{1}+e_{1} \eta .
\end{aligned}
$$

With a sophisticated iteration procedure, they cancelled out high-order terms in $1 / \operatorname{Re}_{\tau} \sim 1 / y^{+} \sim \eta$ and produce a so-called generalized log-law form, with a large number of coefficients to be determined. Although the expansion seems to extend the domain of description and improves the prediction accuracy on Karman coefficient including the $R e$-effect, their analysis, in the absence of any physical explanation, seems not very helpful. It is also unclear what values these coefficients have in pipe flow or boundary layer.

While the above discussions focus on the structure of expansion, others attempt to invoke invariance principles. Barenblatt and co-workers [17,18] proposed a concept of incomplete similarity for large $R e$-limit. It begins with the dimensionless relationship for the mean-velocity gradient,

$\Pi=y^{+} \frac{\partial U^{+}}{\partial y^{+}}=f\left(y^{+}, R e\right)$.

In the limit $v \rightarrow 0$, the complete similarity is

$y^{+} \frac{\partial U^{+}}{\partial y^{+}}=f(\infty, \infty)=$ const $=\frac{1}{\kappa}$,

which indicates the log-law. However, they assumed an incomplete similarity as

$y^{+} \frac{\partial U^{+}}{\partial y^{+}}=g(R e) y^{+\alpha(R e)}$,

which gives a power law scaling, with some supporting observations [19].

According to the Lie group theory, Oberlack [20,21] made a "maximum symmetry" assumption to determine a unique transformation of all variables for parallel flows. This transformation maintains four kinds of invariant scaling for $U^{+}$ versus $y^{+}$, namely, "linear-linear" scaling identified in the sub-layer; "linear-log" and "log-log" scaling corresponding to log-law and power law, respectively; and a newly predicted "log-linear" scaling in the wake. Since all these local scalings 
are only for parts of the whole profile, a consistent composite form (valid for the whole domain and for finite $R e$ ) is required.

Note that all the above theories relate the velocity profile to invariance principles in an "ad hoc" way, without any sound physical justification. This is why the state-of-the-art involves many stark controversies, some of which should be resolved with more dynamical information on turbulence. After fluctuation structures are taken into account, refined features of the velocity profile will be revealed and methods of selecting appropriate invariance groups may become clear. There is no evidence that the near-asymptotic is unique and hence also the log-law. Indeed, we will show that a better alternative exists as we give a wider profile that includes log-region as a sub-domain (see Sect. 4.3).

\subsection{Empirical modeling of mean velocity profile}

The second kind of theory describes the entire mean velocity profile by fitting experimental and numerical results. With the presence of high-quality data due to the progress of measurement and computational technologies, this approach has yielded significant outcomes in recent years, including Nickels's model, MCN model, and L'vov et al. [22] (heretofore referred to as LPR model).

Nickels employed a three layer model, a polynomial description of the sub-layer with exponential modulation, a logarithmic function for the log-layer with high-order modulation, and an exponential decay function for the wake-for mean velocity profiles of TBL, channel and pipe. An interesting feature of his description is a passage from boundary layer to channel with slight modification of the wake function. The model is a parameterization with some effect of pressure gradient taken into account.

The MCN model obtains a two-layer formula for mean velocity profile (only) of TBL at high $R e$, which is a parameterization of a massive set of experimental data (IIT \& KTH data). They fit the log-law indicator function $\Gamma=y^{+} \mathrm{d} U^{+} / \mathrm{d} y^{+}$for the inner layer and the velocity defect $\left(U_{\infty}^{+}-U_{\text {outer }}^{+}\right)$for the outer layer, each yielding a logarithmic form limit. The two layers are hence matched at the overlap region as

$U_{\text {composite }}^{+}=U_{\text {inner }}^{+}+U_{\text {outer }}^{+}-U_{\log }^{+}$.

However, their formulation is rather complicated (with dozens of coefficients), and only valid for relatively high $R e$, rather than finite $R e$. Moreover, its empirical nature results in the inability to describe other flows like channel and pipe flows.

In contrast to Nickels and MCN, the LPR theory proposes an alternative physical model of turbulent mean flow over the whole channel and pipe with a partial inclusion of the properties of the fluctuation field. They introduce the energy balance equation and even (remotely) the Reynolds stress balance equation for defining relevant length scales, giving two unknown functions: one wall function, $r_{\mathrm{W}}$, and one length function, $\ell$. Both are modeled with inspiration from available DNS data.

The weak part of the LPR theory is its intuitive nature, which manifests in three aspects: first its inability in analyzing and improving the degree of approximation; second, the form of the two critical functions determining the mean profile, $r_{\mathrm{W}}$ and $\ell$, appears to be arbitrary; and finally, the LPR theory, does not contain a theoretical framework which allows a possible extension to boundary layer or other flows.

\subsection{Challenge}

The log-law versus power-law controversies [23-26] have attracted a great deal of attention in recent years, but the debate may be interpreted differently. From a mathematical point of view, either log-law or power-law could be a leadingorder approximation at an infinite $R e$, depending on which invariance principle (matching) is used in the derivation. On the other hand, Oberlack and Rosteck [27] pointed out that basic invariance principle is valid in only a restricted domain of the flow, and there may be many other locally valid expressions. Since the asymptotic form in any restricted zone is not unique, fighting over log-law or power-law seems futile. Instead, it is more important to find an approximation which works more accurately at a finite $R e$.

Another major shortcoming of the existing closure theories is that their discussion is limited to the mean velocity profile and the associated mean momentum balance, but hardly involves fluctuation structures. However, studies of turbulent structures during the past several decades have produced strong evidence of the non-trivial role of fluctuations in the mean. In a recent review article, Marusic et al. [28] point out that it is more promising to move toward theories that incorporate fluctuation statistics rather than mean. Hence, constructing a closure description using kinetic energy is the next challenge.

In summary, we believe that the key challenge for wall turbulence is to construct a finite $R e$ theory of the mean quantities (beyond mean velocity profile) for the entire flow domain, from the wall to the outer region. This is what we present now.

\section{SED as a closure theory for general non-equilibrium systems}

\subsection{Two types of complexity}

There are two types of complexity in fluid turbulence: multi-degree of freedom, and multi-factors from different 
environments like wall, compressibility, pressure gradient, buoyancy, rotation, etc. The progress in computational technology has enabled accurate calculation of some simple flows at restricted sets of physical parameters such as $R e$ and Mach number $(M)$, but computation of real engineering flows is generally too costly. Extrapolating computational solutions to realistic physical conditions represents a major challenge owing to the lack of a viable theory. Secondly, the complexity in environments potentially challenges the concept of "universality". There is no unified approach yet to address these complexities.

\subsection{Multiple states for non-equilibrium open systems}

The complexity in fluid turbulence also manifests in the form of a variety of flow structures which display intermittent motions in space and time. When a statistically stationary state exists, velocity and other fluctuations are said to be "self-organized", when a well-behaved probability distribution function exists and hence a set of well-defined mean quantities and root-means-square (rms) fluctuation intensities. Indeed, all stationary processes are self-organized, but complex self-organized turbulent flows are, in addition, characterized by smooth variations of the statistical means and rms intensities in space. We believe that the complexity would manifest in spatial variations which display possibly many, but finite number of, distinct patterns. This thought can be summarized as Multi-state hypothesis: the non-equilibrium turbulent flow generally possesses a finite number of statistical states, and the complexity of the system manifests in the co-existence of multiple states in the form of different layers.

Under this hypothesis, a key question to answer before deriving a viable mean-field theory of turbulence is: How to determine statistical states and what are universal properties of the transitions?

The statistical states should flow from first principles such as conservations of mass, momentum and energy. In the case of turbulent flow, the Navier-Stokes equations govern the space-time variation of fluctuating density, velocity and temperature. Statistical states are thus determined by the EANS equations-the universal balance principles. The difficulty is that the EANS equations are not closed; for example, in the case of incompressible flow, second-order correlation functions appear in the mean momentum equation, third-order correlation functions appear in the equations for the second-order correlation and mean kinetic energy, and so on. This is the notorious closure problem of turbulence. After many decades of research, it is fair to conclude that the traditional approach of adopting "ad hoc" closure assumptions may not be the right way. One needs to find alternatives!

\subsection{SED as a new theoretical framework}

The structure ensemble dynamics (SED) attempts to solve the unclosed EANS equations connecting the mean and fluctuation quantities in the following way. First, it treats the EANS equations as an open system, for which different kinds of closure schema can be permitted for different flow systems or environments. So, it does not forcefully close the EANS equations with a unique closure assumption. Secondly, the multi-state assumption implies that different flow systems are characterized by a distinct set of multi-layer structures. The SED injects the concept of "order functions" (discussed below), for representing the distinct multi-states and the transitions between them. Thirdly, after the order functions are known, the EANS equations fully determine (algebraically) the mean quantities.

Thus, an initial phase of study involves the determination of order functions from empirical (DNS or LES) data. Then the study of order functions using numerical data offers a guide for DNS (LES) study of turbulence. Later, as the properties of the order functions are interpreted in terms of symmetry and other theoretical concepts, the SED approach would form a comprehensive theory for describing the mean quantities and the effects of fluctuation structures on the means.

The SED theory emphasizes three aspects. First, we assume the existence of an ensemble or statistical stationarity. The existence of an ensemble has been inspired by the discovery of the She-Leveque scaling form [29] and explicitly postulated recently [30], which implies that the mean is well-defined, together with the second moment (rms) of fluctuations, and shows smooth behavior with $R e, M$, etc. The turbulence ensemble unambiguously evaluates statistical averages; and the existence of a solution to EANS equations and of a set of order functions are implied. These states are quantitatively described by the balance terms in the mean momentum and energy equations. Second, the goal of SED is to describe the statistical effects of fluctuating structures, which manifest both in smooth spatial variations of the order functions and in their variations with $R e$ and other physical parameters. This is the notion of so-called statistical structure, in terms of multi-states (multi-layers for wall turbulence), which is quite different from geometrical considerations focusing on analyzing instantaneous fields. Theory of statistical structures will involve concepts from group analysis. Third, the word "dynamics" aims at incorporating various physical effects (buoyancy, rotation, roughness, pressure gradients, etc.) which act necessarily in a dynamical way in a non-equilibrium system. Keeping dynamics in mind prevents over-simplified assumptions about turbulent flows; an example of such over-simplification is the mixing length or eddy viscosity approach. The SED searches for all relevant dynamical mechanisms in physical and parameter space by dealing 
with all terms in EANS equations. Although we initially consider the statistically steady situation, it can address active development such as transition to turbulence and unsteady motions.

The three words (ensemble, structure, and dynamics) at this stage appear to be abstract, but they lay down a theoretical framework for pursuing a viable theory of turbulence, capable of dealing with the two kinds of complexity described above. Its initial aim is to produce a quantitative theory of turbulent boundary layer and then a theory for complex flows.

\subsection{Order functions}

Since multiple statistical states exist in physical and parameter spaces, transitions between the states are the key promises of the theory. In statistical physics, the order parameter is a concept to describe a transition (in the parameter space). We thus propose a similar concept - order function-whose spatial variations describe transitions between different statistical states in different flow regimes or layers.

At this stage, we define three types of order functions. The first type is a sensitive indicator, defined by the defect form of the terms in EANS equations, such as $1-S^{+}$where $S^{+}$is the normalized mean shear. They can reveal effects of turbulent structures and can be used to evaluate the accuracy and consistency of numerical simulations, because their near-wall or central asymptotic behaviors can be analytically obtained. The second is a ratio of any two terms in the EANS equations, representing different physical actions. This ratio describes the relative importance of the two actions and hence physical mechanisms. Locations where specific action terms crossover are important information of statistical dynamics. A typical example is the ratio of production to dissipation. The third type is scaling function defined by dimensional analysis. They determine the scaling of the terms in the balance equations, and hence the dependence on $R e$ and other physical parameters. A typical case is the mixing length $\ell_{\mathrm{m}}$, which shows a linear scaling in the logarithmic region.

Generally speaking, the order functions describe the variation of statistical states in space, being effects of fluctuations on the mean. We keep the specific definition of order function open and do not exhaust all order functions here, as additional observations may reveal new order functions. However, how order functions depend on the details of the flows is unclear yet. We speculate that, as $R e$ increases, they will exhibit important universal properties, some of which may even be valid for other flows.

\subsection{Multi-layer hypothesis}

A multi-layer hypothesis is specifically proposed for wall turbulence, as derived from multiple states for general non-equilibrium systems. Specifically, we assume that the ensemble of fluctuating structures displays a finite number of statistical states, depending on the distance from the wall; these states define the multi-layer structures as seen in order functions, resulting in a local similarity statement

$\frac{\phi^{s}}{\phi_{0}^{s}}=h^{s}\left(\frac{y}{y_{0}^{s}}\right)$,

where $s$ donates each local domain, and $\phi$ denotes the order function. $\phi_{0}^{s}$ and $y_{0}^{s}$ are the local scalings. The multi-layer hypothesis suggests that $s>1$, and the spatial variation of $\phi$ involves multiple transitions, from one layer to another. A complete description across the entire domain requires a composite expression discussed in Sect. 4.3 below.

In summary, the SED suggests development of a more complete multi-layer description to incorporate all dynamical features at both the momentum and the kinetic energy balance levels. Traditional two-layer (sub-layer and wake) or three-layer (sub-layer, log-layer and wake) model, as a beginning of this description, remains at the empirical level and applies to the mean velocity only. A composite expression based on invariance principles, especially for the entire domain and finite $R e$, is needed.

\section{SED closure theory for wall-bounded turbulence}

In this section, we present the SED closure theory for wallbounded turbulence, including universal balance principles (the EANS equations) and the order functions, and a quantitative modeling using hyperbolic transition functions.

\subsection{Universal balance principles}

The ensemble averaged mass and momentum equations are

$\frac{\partial \bar{\rho}}{\partial t}+\frac{\partial \overline{\rho u_{j}}}{\partial x_{j}}=0$,

$\frac{\partial \overline{\rho u_{i}}}{\partial t}+\frac{\partial \overline{\rho u_{i} u_{j}}}{\partial x_{j}}+\frac{\partial \bar{p}}{\partial x_{i}}=\frac{\partial \overline{t_{i j}}}{\partial x_{j}}$,

where

$t_{i j}=-\frac{2}{3} \mu \frac{\partial u_{k}}{\partial x_{k}} \delta_{i j}+\mu\left(\frac{\partial u_{i}}{\partial x_{j}}+\frac{\partial u_{j}}{\partial x_{i}}\right)$.

Consider wall-bounded stationary turbulent flow with two inhomogeneous directions: $x$ streamwise and $y$ wall-normal. Then, the streamwise momentum equation is

$$
\frac{\partial \bar{p}}{\partial x}=\left(\frac{\partial \overline{\mu \frac{\partial u}{\partial y}}}{\partial y}+\frac{\partial \overline{\mu \frac{\partial v}{\partial x}}}{\partial y}+\frac{\partial \overline{\bar{t}_{11}}}{\partial x}\right)-\left(\frac{\partial \overline{\rho u u}}{\partial x}+\frac{\partial \overline{\rho u v}}{\partial y}\right) .
$$


Integrate Eq. 15 over $y$, we obtain

$\tau=S+W$,

where $\tau$ is the total stress, $S$ is the shear stress and $W$ includes all nonlinear terms defined below. For convenience, we make the following decomposition

$\tau=\tau_{\mathrm{w}}-\tau_{\mathrm{p}}$,

$S=S^{\perp}+S^{/ /}$,

$W=W^{\perp}+W^{/ /}$,

where $\tau_{\mathrm{w}}=\left.\overline{\mu \frac{\partial u}{\partial y}}\right|_{y=0}$ is the wall shear stress and $\tau_{\mathrm{p}}=$ $-\int_{0}^{y} \frac{\partial \bar{p}}{\partial x} \mathrm{~d} y$ is the stress defect; $\perp$ and // indicate effect due to variations in the normal and streamwise directions, respectively

$S^{\perp}=\overline{\mu \frac{\partial u}{\partial y}}$

$S^{/ /}=\overline{\mu \frac{\partial v}{\partial x}}+\int_{0}^{y} \frac{\partial \overline{t_{11}}}{\partial x} \mathrm{~d} y$,

$W^{\perp}=-\overline{\rho u v}$,

$W^{/ /}=-\int_{0}^{y} \frac{\partial \overline{\rho u u}}{\partial x} \mathrm{~d} y$.

Under the Reynolds decomposition defined by

$u=U+u^{\prime}, \quad v=V+v^{\prime}, \quad w=W+w^{\prime}$,

$p=p_{0}+p^{\prime}, \quad \mu=\mu_{0}+\mu^{\prime}, \quad \rho=\rho_{0}+\rho^{\prime}$,

we obtain

$$
\begin{aligned}
\tau= & S_{0}^{\perp}+S_{0}^{/ /}+S_{2}^{\perp}+S_{2}^{/ /}+W_{0}^{\perp}+W_{0}^{/ /} \\
& +W_{2}^{\perp}+W_{2}^{/ /}+W_{3}^{\perp}+W_{3}^{/ /},
\end{aligned}
$$

where

$$
\begin{aligned}
& S_{0}^{\perp}=\mu_{0} \frac{\partial U}{\partial y}, \\
& S_{0}^{/ /}=\mu_{0} \frac{\partial V}{\partial x}+\frac{2}{3} \partial_{x} \int_{0}^{y}\left(2 \mu_{0} \frac{\partial U}{\partial x}-\mu_{0} \frac{\partial V}{\partial y}\right) \mathrm{d} y, \\
& S_{2}^{\perp}=\overline{\mu^{\prime} \frac{\partial u^{\prime}}{\partial y}}, \\
& S_{2}^{/ /}=\overline{\mu^{\prime} \frac{\partial v^{\prime}}{\partial x}}+\frac{2}{3} \partial_{x} \int_{0}^{y}\left(2 \overline{\mu^{\prime} \frac{\partial u^{\prime}}{\partial x}}-\overline{\mu^{\prime} \frac{\partial v^{\prime}}{\partial y}}-\overline{\mu^{\prime} \frac{\partial w^{\prime}}{\partial z}}\right) \mathrm{d} y, \\
& W_{0}^{\perp}=-\rho_{0} U V, \\
& W_{0}^{/ /}=-\frac{\partial}{\partial x} \int_{0}^{y} \rho_{0} U U \mathrm{~d} y,
\end{aligned}
$$

$$
\begin{aligned}
& W_{2}^{\perp}=-\left(\rho_{0} \overline{u^{\prime} v^{\prime}}+U \overline{\rho^{\prime} v^{\prime}}+V \overline{\rho^{\prime} u^{\prime}}\right), \\
& W_{2}^{\prime /}=-\frac{\partial}{\partial x} \int_{0}^{y}\left(\rho_{0} \overline{u^{\prime} u^{\prime}}+2 U \overline{\rho^{\prime} u^{\prime}}\right) \mathrm{d} y, \\
& W_{3}^{\perp}=-\overline{\rho^{\prime} u^{\prime} v^{\prime}}, \\
& W_{3}^{\prime /}=-\frac{\partial}{\partial x} \int_{0}^{y} \overline{\rho^{\prime} u^{\prime} u^{\prime}} \mathrm{d} y .
\end{aligned}
$$

The subscript indicates the order of the correlation of fluctuation terms. Note that for the incompressible channel flow, $S=S_{0}^{\perp}$ and $W=W_{2}^{\perp}$.

Denoting the ensemble averaged density and viscosity at the wall as $\rho_{\mathrm{w}}$ and $v_{\mathrm{w}}$, respectively, the wall scales, namely the friction velocity $u_{\tau}$ and the viscous length scale $\ell_{\mathrm{S}}$, are

$u_{\tau} \equiv \sqrt{\frac{\tau_{\mathrm{w}}}{\rho_{\mathrm{w}}}}, \quad \ell_{\mathrm{s}} \equiv \frac{v_{\mathrm{w}}}{u_{\tau}}$.

Hence, the dimensionless variables are

$y^{+} \equiv \frac{y}{\ell_{\mathrm{s}}}, \quad u^{+} \equiv \frac{u}{u_{\tau}}, \quad \mu^{+} \equiv \frac{\mu}{\rho_{\mathrm{w}} \nu_{\mathrm{w}}}$.

Note that for the channel and pipe flow, the dimensionless outer length scale $\eta$ is $y / \Delta$ where $\Delta$ is the half height of the channel or the radius of the pipe. In the following sections, we also use the center length scale

$z \equiv 1-\eta$,

which represents the normalized distance from the center line in the channel or pipe.

The mean momentum equation (21) can be normalized by dividing $\rho_{\mathrm{w}} u_{\tau}^{2}$. Inspecting the magnitude and keeping the leading terms, we obtain an approximate balance equation (also confirmed by DNS)

$\left(S_{0}^{\perp}\right)^{+}+\left(W_{0}^{\perp}\right)^{+}+\left(W_{0}^{/ /}\right)^{+}+\left(W_{2}^{\perp}\right)^{+}+\tau_{\mathrm{p}}^{+} \approx 1$.

Note that the mean momentum equation (21) takes a universal form though differing in actual functional forms for different flows. This manifests in the study of incompressible versus compressible boundary layers.

Under the Favre decomposition $\left(g=\widetilde{g}+g^{\prime \prime}\right)$, we have a set of slightly different expressions

$$
\begin{gathered}
S_{0}^{\perp}=\overline{\mu \frac{\partial u}{\partial y}}, \quad S_{0}^{/ /}=\overline{\mu \frac{\partial v}{\partial x}}+\frac{\partial}{\partial x} \int_{0}^{y} \overline{t_{11}} \mathrm{~d} y, \\
W_{0}^{\perp}=-\bar{\rho} \tilde{u} \widetilde{v}, \quad W_{0}^{/ /}=-\frac{\partial}{\partial x} \int_{0}^{y} \bar{\rho} \tilde{u} \widetilde{u} \mathrm{~d} y, \\
W_{2}^{\perp}=-\overline{\rho u^{\prime \prime} v^{\prime \prime}}, \quad W_{2}^{/ /}=-\frac{\partial}{\partial x} \int_{0}^{y} \overline{\rho u^{\prime \prime} u^{\prime \prime}} \mathrm{d} y .
\end{gathered}
$$


The equation for the ensemble averaged kinetic energy $\overline{\rho k}=\frac{1}{2} \overline{\rho u_{i}^{\prime \prime} u_{i}^{\prime \prime}}$, is a balance among energy production $P$, viscous dissipation $\varepsilon$, turbulent transport $T$, convection $C$, and pressure transport $\Pi$

$P+T+C+\Pi=\varepsilon$,

where

$P=-\overline{\rho u_{i}^{\prime \prime} u_{j}^{\prime \prime}} \frac{\partial \tilde{u}_{i}}{\partial x_{j}}$,

$\varepsilon=-\frac{\partial \overline{u_{i}^{\prime \prime} t_{i j}^{\prime}}}{\partial x_{j}}-\overline{u_{i}^{\prime \prime}} \frac{\partial \overline{t_{i j}}}{\partial x_{j}}+\overline{t_{i j}^{\prime} \frac{\partial u_{i}^{\prime \prime}}{\partial x_{j}},}$

$T=-\frac{1}{2} \frac{\partial \overline{\rho u_{i}^{\prime \prime} u_{i}^{\prime \prime} u_{j}^{\prime \prime}}}{\partial x_{j}}$

$C=-\frac{\partial\left(\overline{\rho k} \widetilde{u_{j}}\right)}{\partial x_{j}}$,

$\Pi=-\frac{\partial \overline{p^{\prime} u_{j}^{\prime \prime}}}{\partial x_{j}}-\overline{u_{j}^{\prime \prime}} \frac{\partial \bar{p}}{\partial x_{j}}+\overline{p^{\prime} \frac{\partial u_{j}^{\prime \prime}}{\partial x_{j}}}$.

For incompressible flows, $k \equiv\left(\overline{u^{\prime 2}}+\overline{v^{\prime 2}}+\overline{w^{\prime 2}}\right) / 2$ and the equation terms are

$P=-\overline{u_{i}^{\prime} u_{j}^{\prime}} \frac{\partial U_{i}}{\partial x_{j}}, \quad \varepsilon=-v \frac{\partial^{2} k}{\partial x_{j} \partial x_{j}}+v \overline{\frac{\partial u_{i}^{\prime}}{\partial x_{j}} \frac{\partial u_{i}^{\prime}}{\partial x_{j}}}$,

$T=-\frac{1}{2} \frac{\partial \overline{u_{i}^{\prime} u_{i}^{\prime} u_{j}^{\prime}}}{\partial x_{j}}, \quad C=-\frac{\partial k U_{j}}{\partial x_{j}}$,

$\Pi=-\frac{1}{\rho} \frac{\partial \overline{p^{\prime} u_{j}^{\prime}}}{\partial x_{j}}$.

The kinetic energy equations (29) and (30) can also be normalized in wall units, denoted by + . With this notation, the order functions are similar in incompressible and compressible flows.

4.2 Order functions and a multi-layer picture of wall turbulence

Here, we define a set of order functions, specific to the study of wall-bounded turbulent flows. Note that the theoretical framework is universally applicable to incompressible and compressible flows, although their concrete physics are different. What follows illustrates the application of the concept to an incompressible turbulent channel flow and a compressible turbulent boundary layer, which clearly reveals a multilayer picture of wall turbulence, and which then motivates a quantitative model for their spatial variations in the next subsection. The results support the validity of a procedure for systematically extracting quantitative information from DNS data.

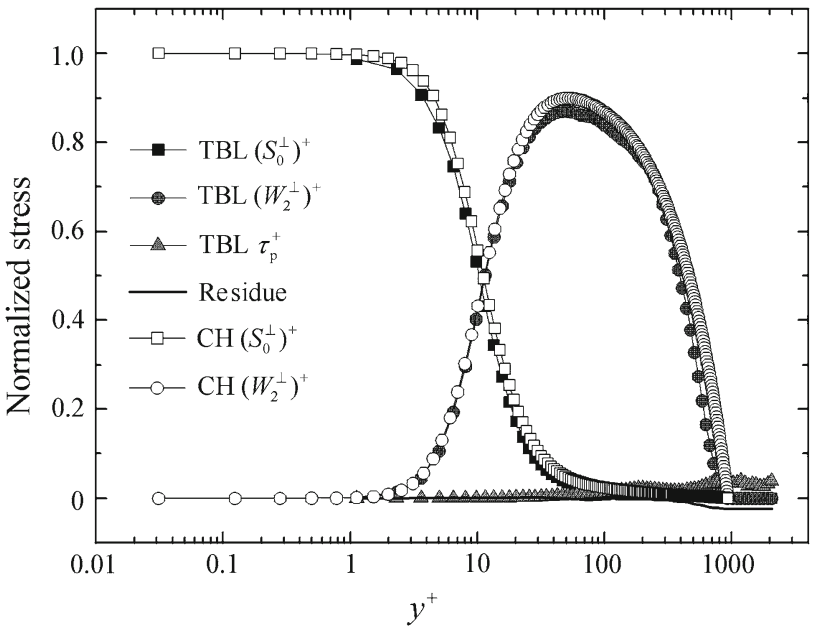

Fig. 1 Variation of the shear stress and Reynolds stress for the incompressible channel flow and compressible TBL, showing three sections: near the wall $\left(y^{+}<1\right)$, the transition region $\left(1<y^{+}<40\right)$ and the outer region $\left(y^{+}>40\right)$ at the mean momentum level. The residue counts for the contribution of all the neglected terms in Eq. 26. Simulation data are from Jimenez et al. [31] $\left(R e_{\tau}=950\right)$ and Li et al. [32] $(M=$ $2.25, \operatorname{Re}_{\theta}=4,000$, under the isothermal boundary condition at the wall)

\subsubsection{Sensitive indicators $\tau_{\mathrm{d}}^{+}$and $S_{\mathrm{d}}^{+}$}

The first type of order functions, namely sensitive indicators, are defined in the defect form-for example $\tau_{\mathrm{d}}^{+} \equiv 1-\tau^{+}$ and $S_{\mathrm{d}}^{+} \equiv 1-\left(S_{0}^{\perp}\right)^{+}$. The two functions are important to present a global structure of the flow.

Figure 1 shows the stress distribution (including shear stress and Reynolds stress) for an incompressible channel and a compressible boundary layer. The two systems show the similar global structure at the mean momentum level (when Favre decomposition is used for the compressible boundary layer), with slight quantitative difference in the outer regions, as expected. The SED analysis will pay more attention to such quantitative aspect, and detailed results will be reported later.

An interesting aspect of the sensitive indicators is their leading asymptotic behavior in Eq. 16, which may indicate the computational accuracy of DNS or LES. This is discussed in Sect. 5.2.

\subsubsection{Ratio functions $\sigma, v_{t}^{+}$and $\Theta_{v}$}

Below, we discuss three order functions of the second type, in the ratio form. Following Eq. 26,

$\sigma \equiv \frac{\left(W_{2}^{\perp}\right)^{+}}{S_{\mathrm{d}}^{+}}$

we characterizes the importance of turbulent fluctuations (Reynolds stress) relative to mean viscous shear. As shown in Fig. 2, a three-layer picture is seen for the channel flow: for 


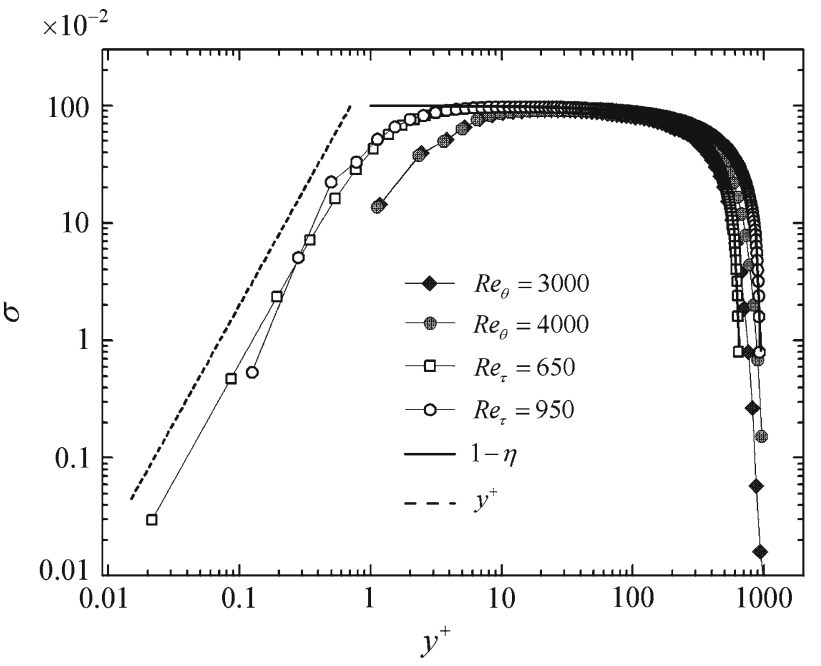

Fig. 2 The stress ratio $\sigma$ in an incompressible channel flow and a compressible TBL $(M=2.25)$, suggesting a two-layer picture at the mean momentum level for the former and a multi-layer for the latter. Simulation data are from Iwamoto et al. [33] $\left(R e_{\tau}=650\right)$, Jimenez et al. [31] $\left(R e_{\tau}=950\right)$ and Li et al. [32] $(M=2.25)$

$y^{+}<1$, an asymptotic $y^{+2}$ is present due to effect of constant pressure gradient near the wall; for $y^{+} \approx 10, \sigma \sim 1$; and for $y^{+}>40, \sigma \approx 1-\eta$ due to the global stress $\tau_{\mathrm{p}}^{+}=\eta$. On the other hand, the compressible TBL has a similar plateau $(\sigma \approx 1)$ near $y^{+}=10$, with similar fall-offs both towards the wall and the outer region. Clearly, the outer-layer fall-off has a $R e$-dependence which needs to be characterized in detail. Note that the nature of the fall-off towards the wall is also interesting, because for the zero-pressure-gradient incompressible boundary layer, one expects that the plateau $\sigma=1$ extends all the way to the wall, since $\tau_{\mathrm{p}}$ is very small and can be neglected. The current SED analysis raises a question: is it possible that the compressible TBL at $M=2.25$ produces an effective pressure gradient in the flow? Results of this study will be reported later.

The widely used eddy viscosity turns out to be also a ratio function. Following Eq. 26,

$v_{t}^{+} \equiv \frac{\left(W_{2}^{\perp}\right)^{+}}{\left(S_{0}^{\perp}\right)^{+}}$.

Detailed studies of this order function will be reported elsewhere.

Note that Eq. 32 is a normalized version of the usual eddyviscosity. In fact, in the SED definition of order functions, we frequently make dimension-like arguments on quantities normalized using the wall shear stress or wall velocity. The reason is that we attempt to extract additional symmetry properties beyond elementary dimensional transformation, which are important to govern the rules behind spatial variations. A complete theoretical justification for this has to await a

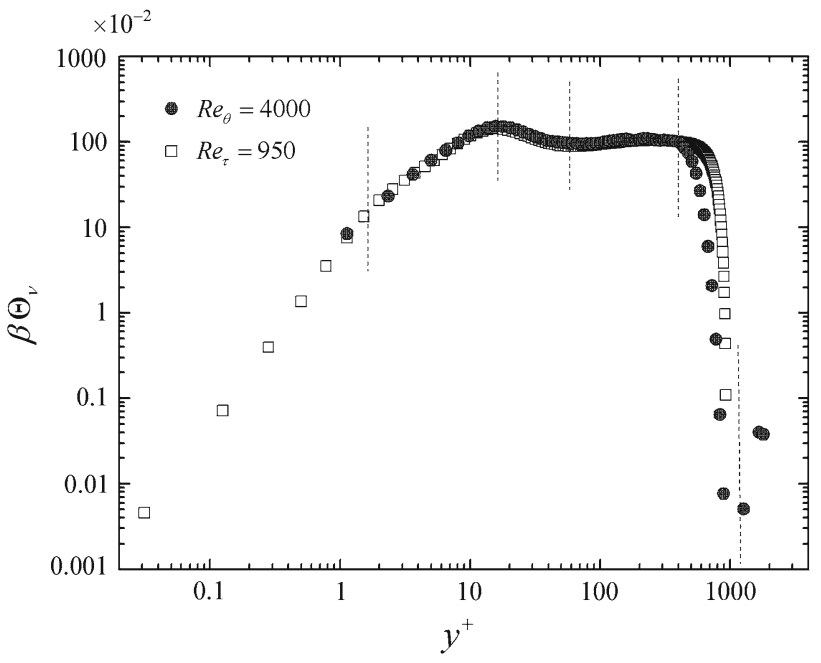

Fig. $3 \beta \Theta_{v}=\beta P^{+} / \epsilon^{+}=\left(S_{0}^{\perp}\right)^{+}\left(W_{2}^{\perp}\right)^{+} / \epsilon^{+}$reveals multi-layers in the kinetic energy equation. Data are the same as in Fig. 2

Lie-group analysis of the Navier-Stokes equations, to be reported elsewhere.

Finally, we report a ratio function which is most relevant to the energy dynamics, namely the ratio of turbulent production $P^{+}$to dissipation $\epsilon^{+}$

$\Theta_{v} \equiv \frac{P^{+}}{\epsilon^{+}}$,

which represents the main balance in the fluctuations. This function reveals a new set of layers in EANS equations, as fluctuation structures are key to energy dissipation. For the compressible flow, we define a ratio factor $\beta$ to describe the relation between the mean shear and Reynolds stress with the turbulent production term in the kinetic energy equation

$\beta\left(y^{+}\right) \equiv \frac{\left(S_{0}^{\perp}\right)^{+}\left(W_{2}^{\perp}\right)^{+}}{P^{+}}$.

It equals unity in the incompressible flow and is a function of $y^{+}$in the compressible flow.

Figure 3 shows five layers in $\beta \Theta_{v}$ in the channel flow, transiting at $y^{+}=2,15,45$; outside $y^{+}=45$, the "quasiequilibrium" region $\Theta_{v} \approx 1$ begins; $\Theta_{v}$ eventually goes to zero. It is remarkable that the compressible TBL shows multilayer structures similar to the channel flow, which are even quantitatively very close to each other. The quantification of these layers are critical to a more accurate description of the velocity, Reynolds stress distribution of the wall turbulence, as will be reported later.

\subsubsection{Scaling functions $\ell_{\mathrm{m}}^{+}$and $\ell_{v}^{+}$}

The third type of order function, i.e. scaling function, will be introduced for incompressible flow first, and then extended to compressible flow. 
On the mean momentum level, the mean shear and Reynolds stress are the most important physical effects. The only dimensional expression for a length function with the two quantities is the mixing length

$\ell_{\mathrm{m}}^{+} \equiv \frac{\sqrt{\left(W_{2}^{\perp}\right)^{+}}}{\left(S_{0}^{\perp}\right)^{+}}$.

A more interesting length function involves energy dynamics, in particular, the viscous dissipation $\epsilon^{+}$. Considering a dimensional expression for the dissipation $\epsilon^{+}$in terms of $\left(S_{0}^{\perp}\right)^{+},\left(W_{2}^{\perp}\right)^{+}$, and a length $\ell^{+}$, a general form is

$\epsilon^{+}=\left(W_{2}^{\perp}\right)^{+(1+n / 2)}\left(S_{0}^{\perp}\right)^{{ }^{(1-n)}} \ell^{+(-n)}$,

where $n$ is an arbitrary integer. Note that as $n \rightarrow \infty$, the dissipative contribution vanishes and we recover the mixing length $\ell_{\mathrm{m}}^{+}$. Inspecting DNS data of incompressible channel flow, we found that $\ell_{\mathrm{m}}^{+}$and other choices of $n$ show a divergence at the channel center. If and only if $n=4$, does $\ell^{+}$ saturate to a nonzero limit which can be analytically proved. Hence we define a characteristic dissipative length scale $\ell_{v}^{+}$

$\ell_{v}^{+}=\left[\frac{\left(W_{2}^{\perp}\right)^{+3}}{\left(S_{0}^{\perp}\right)^{+3}} / \epsilon^{+}\right]^{1 / 4}=\left(v_{t}^{+3} / \epsilon^{+}\right)^{1 / 4}$,

which converges to a constant at the channel center. Indeed, a general relationship between $\ell_{\mathrm{m}}^{+}$and $\ell_{v}^{+}$is

$\ell_{\mathrm{m}}^{+}=\ell_{v}^{+}\left(\beta \Theta_{v}\right)^{1 / 4}$.

Figure 4 shows the variation of $\ell_{v}^{+}$for the two flows. Interestingly, a specific $1-z^{4}$ structure layer is discovered for $\ell_{v}^{+}$in the channel flow. Note that it can be written as $(1-z)\left(1+z+z^{2}+z^{3}\right) \sim y^{+}$, which indicates a consistency of the bulk flow to an universal logarithmic constraint near the wall. On the other hand, we find a critical location for compressible TBL at $y^{+}=800$, which is used to define outer layer location $z_{T}$

$z_{T} \equiv 1-\frac{y^{+}}{800}$

The $z_{T}$ coordinate helps one to identify a similar $1-z_{T}^{3}$ structure in the compressible TBL.

\subsection{Hyperbolic function modeling of multi-layer structures}

The results shown above clearly demonstrate a multi-layer picture of wall turbulence. We now propose a method for its quantitative modeling. The newly generated function has a hyperbolic-like structure, which we will call the SED base function.

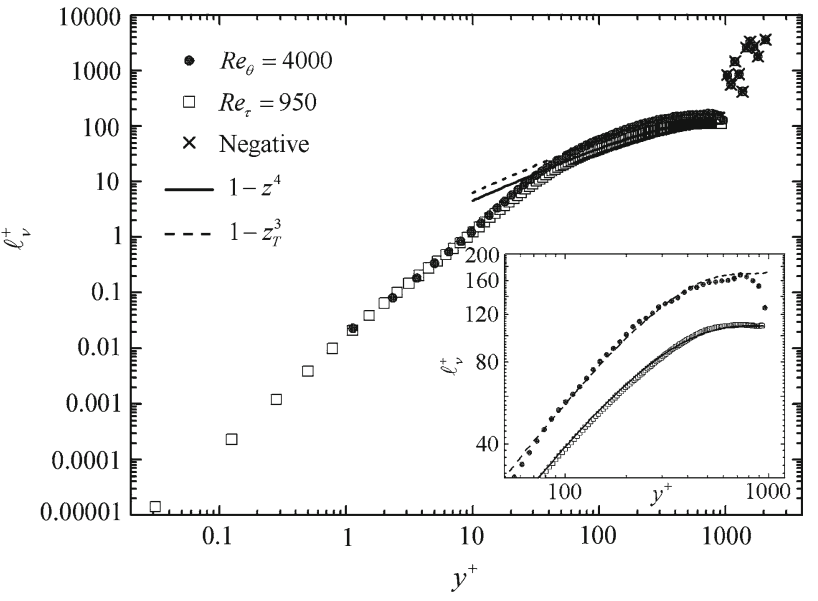

Fig. 4 The eddy dissipation length scale $\ell_{v}^{+}$. Data are the same as in Fig. 2. The two flow systems again show the same structure near the wall, but a slightly different outer-layer structure. The incompressible channel flow shows a $1-z^{4}$ bulk flow structure, and the compressible TBL shows a $1-z_{T}^{3}$ structure

\subsubsection{SED base function}

In Sect. 2.1, it is explained that the log-law or power-law can be derived from a matching (invariance) equation (3) or (4). However, neither log-law nor power-law holds for the entire domain of the flow. A function capable of describing the multi-layer structure must exhibit transitions from one state to another. Therefore, we need to extend current invariance analysis to describe multi-layer structures displayed by the order functions.

In order to permit a transition, we propose a generalized invariance equation

$\frac{y^{1-\beta}}{\left(\phi / y^{\gamma}\right)^{1-\alpha}} \frac{\mathrm{d}\left(\phi / y^{\gamma}\right)}{\mathrm{d} y}=C$,

where $\phi$ denotes an order function, and $\alpha, \beta, \gamma$ and $C$ are parameters. Several special cases are noteworthy. When $\gamma=0, \alpha=1$ and $\beta=0$, Eq. 40 yields a log-law; when $\alpha=0$ and $\beta=0$, it yields a power low; when $\alpha=0, \beta=1$, it yields an exponential solution. These are solutions obtained by Oberlack [21]. However, more general cases involves $\alpha \beta \neq 0$; in this case, direct integration of equation (40) yields a generalized solution

$\phi=c_{1} y^{\gamma}\left(1+\frac{y^{\beta}}{c_{2}}\right)^{1 / \alpha}$,

where $c_{1}$ and $\gamma$ are parameters and $c_{2}=c_{1}^{\alpha} \beta /(C \alpha)$. The formula (41) indicates that $\phi$ displays a transition from one power-law state of exponent $\gamma$, to another state of exponent $\gamma+\beta / \alpha$, as $y$ varies from zero to infinity. Hence, the generalized invariance equation (40) is capable of describing a two-state with a transition. We can rewrite Eq. 41 in the form 
of a hyperbolic-like equation for $y$ and $\phi$

$\left(\frac{\phi}{c_{1} y^{\gamma}}\right)^{\alpha}-\frac{y^{\beta}}{c_{2}}=1$,

which may be important for the discussion of the geometry of invariant surfaces related to multi-layer structures. Furthermore, when a series of transitions occur, we simply develop a multiplicative description

$\phi=\phi_{0} \prod_{0}^{n-1}\left[1+\left(\frac{y}{y_{i}}\right)^{p_{i}}\right]^{n_{i} / p_{i}}$,

as a complete prescription for $\phi$ in the entire domain. An interesting feature of Eq. 43 is that it can be approximated by Eq. 41 , when $y$ is near $c_{2}$ (location relevant to a particular layer). This makes Eq. 43 adequate for describing complex multi-layer structures.

The most important part of Eq. 41 is

$\phi=c_{1}\left(1+\frac{y^{\beta}}{c_{2}}\right)^{1 / \alpha}$,

which describes a transition from 1 to a power-law state of exponent $\beta / \alpha$, and is the solution of the following invariance equation

$\frac{y^{1-\beta}}{\phi^{1-\alpha}} \frac{\mathrm{d} \phi}{\mathrm{d} y}=C$.

Because of its importance in describing transition between states, we will call Eq. 44 an SED base function. Note that Eq. 44 has been widely used previously in turbulence research, such as the Batchelor interpolation formula [34], the von Karman energetic-range correction for energy spectrum model [35], the wall function $\tau_{\mathrm{W}}$ in LPR model [22], and so on.

In Fig. 5, we show schematically a function displaying a four-layer structure at critical locations $y^{+}=5$ and 40, and $z=1-y^{+} / R e_{\tau}=0.5$

$$
\begin{aligned}
f\left(y^{+}, z\right)= & y^{+}\left[1+\left(\frac{y^{+}}{5}\right)^{4}\right]^{-2 / 4}\left[1+\left(\frac{y^{+}}{40}\right)\right]^{4 / 4} \\
& \times\left[1+\left(\frac{z}{0.5}\right)^{-4}\right]^{-2 / 4} .
\end{aligned}
$$

This type of function is used for modeling the behavior of the order functions such as $\ell_{\mathrm{m}}^{+}$in the next subsection.

\subsubsection{A multi-layer quantitative model for $\ell_{\mathrm{m}}^{+}$}

To illustrate the use of SED base function for quantitative modeling of order function, we give an expression for $\ell_{\mathrm{m}}^{+}$, in incompressible channel flow, which is derived from

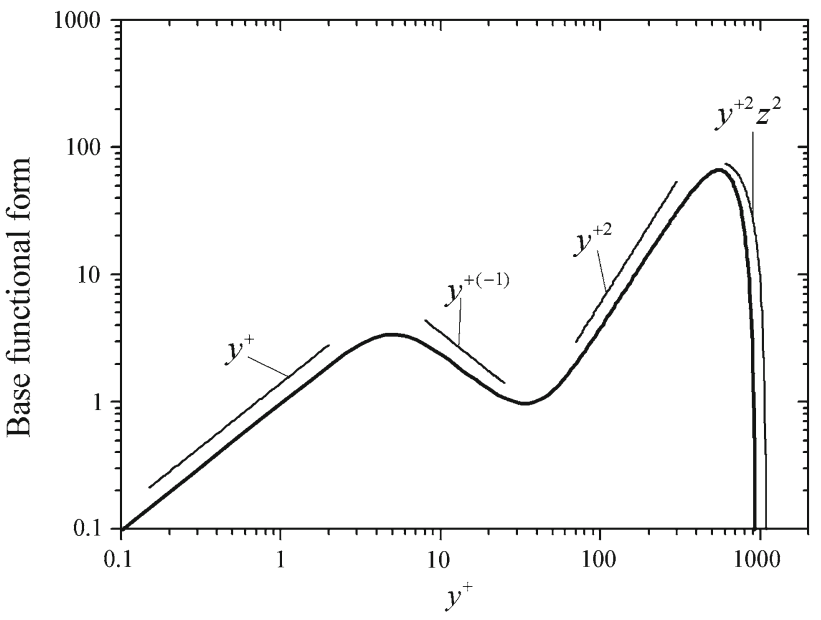

Fig. 5 Schematic of a function displaying a four-layer structure

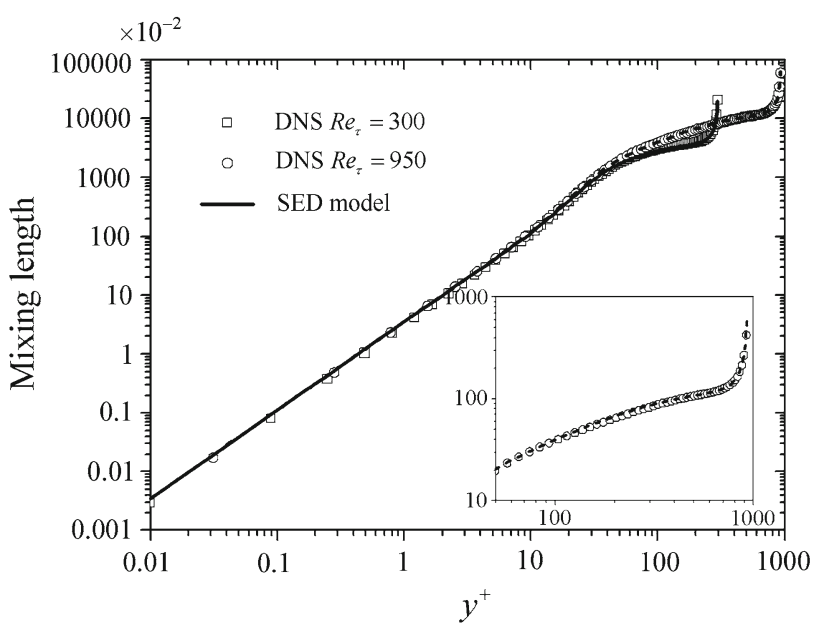

Fig. 6 Comparison of Eq. 47 with DNS data of channel flow, at $R e_{\tau}=$ 300,950 . DNS data are from Iwamoto [33] and Jimenez et al. [31]

inspection of DNS data at several $R e$ [33]

$$
\begin{aligned}
\ell_{\mathrm{m}}^{+}= & \frac{\sqrt{b}}{\sqrt{0.27}}\left(1-z^{4}\right)\left[1+\left(\frac{y^{+}}{10}\right)^{-4}\right]^{1 / 8} \\
& \times\left[1+\left(\frac{y^{+}}{42}\right)^{-6}\right)^{-1 / 6}\left[1+\left(\frac{z}{0.27}\right)^{-5 / 2}\right)^{1 / 5},
\end{aligned}
$$

where $b$ is a limiting value of $\ell_{\mathrm{m}}^{+}$at the center

$b=\lim _{z \rightarrow 0} z\left(W_{2}^{\perp}\right)^{+} /\left[\operatorname{Re}_{\tau}\left(S_{0}^{\perp}\right)^{+}\right]^{2} \approx 0.0036$.

This model shows that the mixing length displays four layers, with three transitions taking place at $y^{+}=10$ where production peaks, $y^{+}=42$ where log-layer begins and $z=$ 0.27 , the crossover of production and turbulent transport.

In Fig. 6, we show the comparison of Eq. 47 with DNS data at several $R e$. The agreement is excellent. Note that for channel flow, knowing $\ell_{\mathrm{m}}^{+}$, the mean momentum balance equation 
fully determines the mean shear, hence the mean velocity profile. Note that this four-layer model is constructed without taking into account the energy dynamics, and a more sophisticated model involving $\ell_{v}^{+}$and $\Theta_{v}$ (using Eq. 38, hence accounting for the energy dynamics) leads to a further improved accuracy. Detailed comparison will be reported elsewhere.

The fact that Eq. 47 is a multiplicative form shows an important feature of the SED modeling: it covers the whole flow domain. A consequence is the ability to predict a link between Karman coefficient (a local property near $y^{+}=$ 45 and the limiting constant $b$ at the channel center. For $y^{+} \approx 45$, Eq. 47 yields, for Karman coefficient $\kappa$, defined as $\ell_{\mathrm{m}}^{+}=\kappa y^{+}$,

$\kappa \approx \frac{\sqrt{b}}{\sqrt{0.27}} \times 4 \times 2^{-1 / 6} \approx 0.41$.

This quantitative agreement strengthens our assertion that the multi-layer picture can give a precise quantitative model which connects the logarithmic law with the bulk structure in the channel.

\section{Application and further development of SED}

\subsection{Building a second-order closure model}

An SED-based second-order closure model is built for incompressible channel flow using four order functions [36]. The first is

$\alpha=\frac{W^{+}}{{\overline{u^{\prime 2}}}^{+}}$,

which was introduced earlier by Bradshaw et al. [37] in an one-equation closure model. The second is an expanded Taylor microscale

$\lambda_{v}^{+2}=\frac{2 \overline{u^{\prime 2}}}{\varepsilon^{+}}$.

The third order function $\lambda_{\mathrm{p}}$ accounts for the fluctuating pressure effects, similar to Taylor scale

$\lambda_{\mathrm{p}}^{+2}=\frac{2 \overline{{u^{\prime 2}}^{+}}}{-\Pi^{+}}$.

Finally, the fourth order function $\beta$ describes the convective effects

$\beta=\frac{\overline{u^{\prime 2} v^{\prime}}}{\overline{u^{\prime 2}}+3 / 2}$.

Given the above four order functions, the momentum and energy equations are closed for the mean velocity $U^{+}$and the

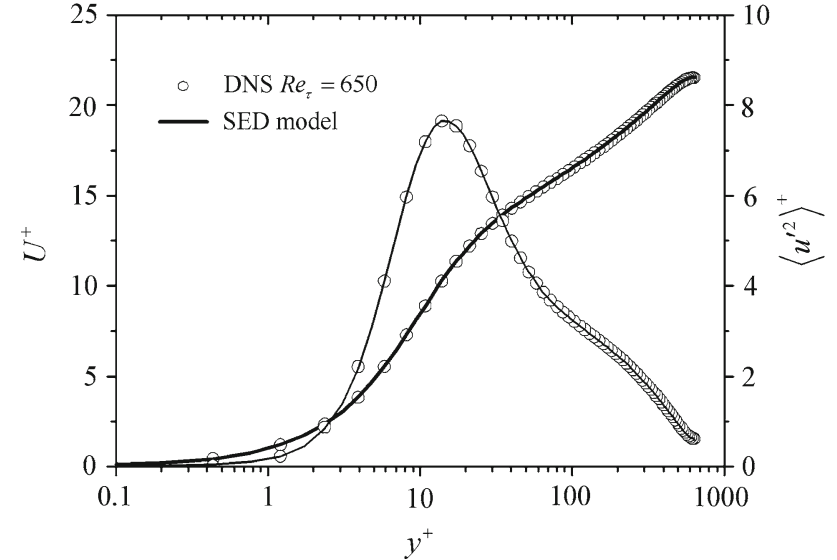

Fig. 7 Steady solution of the SED closure equations for the mean velocity $U^{+}$and kinetic energy starting from zero initial condition, compare with DNS solutions by Iwamoto et al. [33]. Results are taken from Ref. [36]

Table 1 Comparison of wall friction coefficients for a channel flow with $R e_{\tau}=300$ between the predictions of various closure models (from Wilcox [38]) and DNS

\begin{tabular}{lll}
\hline Model & $C_{f} / 10^{-3}$ & Error (\%) \\
\hline DNS & 7.045 & - \\
SED model & 7.026 & -0.26 \\
Stress- $\omega$ model & 7.096 & 0.73 \\
Cebeci-Smith model & 7.365 & -2.8 \\
$k$ - $\omega$ model & 7.254 & 3.0 \\
Baldwin-Lomax model & 6.803 & -3.4 \\
Spalart-Allmaras model & 6.781 & -3.7 \\
Johnson-King model & 6.338 & -10.0 \\
Baldwin-Barth model & 8.007 & 13.7 \\
\hline
\end{tabular}

The SED model shows a significantly higher accuracy. Table is taken from Ref. [36]

mean streamwise kinetic energy $\overline{u^{\prime 2}}$. She et al. [36] demonstrate that this system of equations, starting from zero initial condition, converges to the DNS result with high accuracy in Fig. 7. A further comparison of the friction prediction with various engineering models is presented in Table 1, with SED offering the highest accuracy. Since this result is obtained from order functions determined from the DNS data, such a high accuracy is not unexpected. Nevertheless, this result strongly supports the SED concept.

\subsection{Evaluating numerical simulations}

The SED is a theoretical framework which enables one to evaluate DNS or LES results when analytical results are available asymptotically (near the wall or far from the wall). In this subsection, we introduce a procedure for evaluating the convergence of numerical simulations. This procedure first 


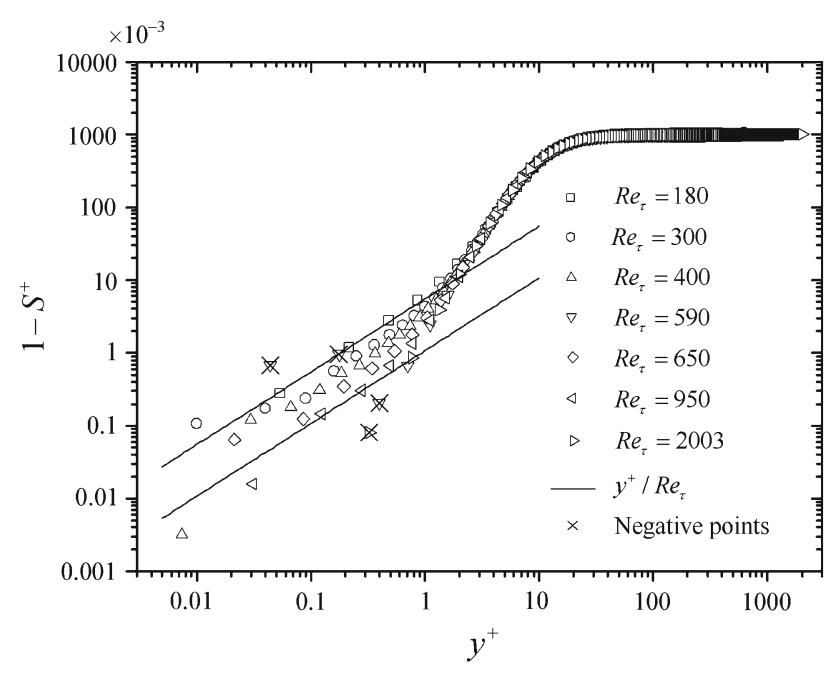

Fig. 8 Defect strain rate $1-S^{+}$derived from DNS data of channel flows at several $R e$ (Moser et al. [39], Iwamoto et al. [33], and Jimenez et al. [31]). Note insufficient near-wall statistical convergence for two data sets of higher $R e_{\tau}$ (Moser, $R e_{\tau}=590$ and Jimenez, $R e_{\tau}=2,003$ )

involves an asymptotic analysis of EANS equations, which must be satisfied by any numerical simulation results. An example is the scaling at the wall and the channel center. We illustrate this procedure by examining a few DNS databases of turbulent channel flow.

Consider the defect stress $S_{\mathrm{d}}^{+}$at the wall. Asymptotic analysis for channel flow yields a $y^{+}$scaling very close to the wall, as a result of the mean pressure gradient $1 / R e_{\tau}$, and a $y^{+3}$ scaling away from the wall due to the contribution of the Reynolds stress $W^{+} \sim y^{+3}$. Figure 8 plots a series of DNS results. Relative to the theoretical constraint, the $R e_{\tau}=590$ data of Moser et al. [39] and $R e_{\tau}=2,003$ data of Jimenez [31] show large scatter near the wall, indicating insufficient resolution or a lack of computational convergence. It is entirely possible that the statistical convergence and the near-wall resolution are not satisfactory. Higher resolution and longer computation seem to be needed.

Another indicator is $z-W^{+}$which is sensitive at the channel center. Asymptotic analysis indicates that $z-W \sim$ $z / R e_{\tau}$, hence plotting $\left(z-W^{+}\right) R e_{\tau}$ for several DNS data (Fig. 9), allows us to verify whether the computation has converged. It is clear from the graph that the $R e_{\tau}=2,003$ data display an anomaly, which appears to be an indication of the lack of statistical convergence in the outer region. Note that the fluctuations $u^{\prime}$ and $v^{\prime}$ do not vanish at the center while $W^{+}$goes to zero. Therefore, the statistical convergence is critical to the accuracy of $W^{+}$. On the other hand, the outer region takes longer to converge, because the turnover time for outer eddies is longer. These two factors combine to increase the difficulty for the center convergence. Nevertheless, several other DNS data seem to have converged, for which the quantitative relation $z-W^{+} \sim z / R e_{\tau}$ seems to be valid.

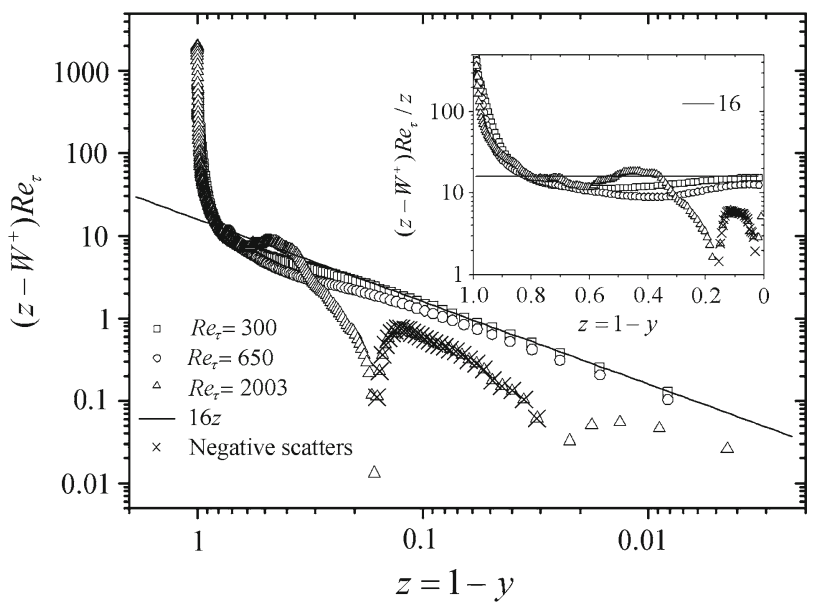

Fig. 9 A sensitive indicator of the central channel dynamics: $\tau^{+}$ $W^{+}=S^{+}$in coordinate $z=1-y^{+} / R e_{\tau}$. Note $R e_{\tau}=2,003$ data exhibit obvious anomaly, due to possibly the lack of statistical convergence, which takes typically longer at the center of the channel and at higher $R e_{\tau}$

Here, we summarize the concrete steps in carrying out an evaluation of simulations (DNS or LES):

1. Compute and examine the spatial variations of order functions, especially the sensitive indicators.

2. Derive asymptotic expression near the wall or other computational boundary, and compare with the simulation data.

3. Compare behavior of the order functions of new simulations with prior simulations in similar regions.

4. Explain the discrepancy in terms of statistical convergence, grid resolution, or other factors.

\subsection{Guiding DNS study of inhomogeneous turbulence}

The SED is also a theoretical framework which enables one to systematically retrieve quantitative information from DNS or LES data. The prevailing closure models (like scaling argument, empirical description of velocity profiles, etc.) offer little guidance for analyzing numerical or experimental data. Typical theoretical measures like energy spectra, correlation functions, skewness, flatness, etc. do not help understanding of the relation between the mean and the fluctuations. In contrast, the order functions in SED promise to reveal this connection, and hence provide a guide to DNS study of wall turbulence.

Here, we summarize the concrete steps in carrying out such a quantitative DNS study:

1. Carry out EANS (balance) equation calculation.

2. Compute relevant order functions to identify multi-layers and dominant actions in each layer. 
3. Identify scaling and transition parameters in each layer and examine their $R e$ dependence.

4. Achieve a quantitative description, using the SED base functions, of a set of mean profiles for a range of $R e$.

5. Develop interpretations of the observed variations of the order functions.

The merit of performing these steps is clear, if the multilayer structure is the essence of turbulent mean flows. We believe that it is not only for canonical channel or boundary-layer flows (as shown above), but also for a variety of wall-bounded turbulent flows in the presence of pressure gradient, roughness, buoyancy, rotation, compressibility, etc. We thus suggest a framework based on accurate description of multi-layer structures, for physical understanding and computational modeling of complex flows. The usefulness of the framework needs to be tested.

Note that the above procedure can be carried out for LES study as well. Although the balance equations may change due to the presence of the subgrid scale term, the way of defining the order functions remains the same. It is particularly interesting to study how some fluctuation budgets are distorted by the subgrid modeling terms, which will then inspire new models for improvement.

\section{Conclusion}

Here, we have established a platform for analyzing the structure of wall-bounded turbulent flows using DNS data. In particular, we have presented a new perspective for deriving quantitative theory of wall-bounded turbulence. Based on a multi-state assumption, we develop a multi-layer picture of wall turbulence, and the SED analysis of DNS data supports its validity. The preliminary results reported above represent the first phase of the SED approach, that is, to use DNS empirical data to evaluate the order functions and to interpret in terms of multi-layer structures. We have also described the beginning of the second phase, namely, using symmetry consideration in forms of generalized invariance equation to develop quantitative models expressing the multi-layer structure. This leads to a new parameterization of empirical data, which is hopefully more accurate since the multi-layer structure is physically closer to the nature of wall turbulence. While details results are deferred to later publications, the theoretical foundation is presented for both incompressible and compressible wall-bounded flows.

Note that the general framework of SED makes two parallel emphases. One is to develop a set of universal concepts adaptable to general non-equilibrium systems (and to offer a solution to the closure problem), and the second is to extract relevant quantitative knowledge from DNS studies.
The progress in the former lays the foundation for the latter. The usefulness of the framework depends critically on the reliability of the DNS data; thus, it is important to apply the theory to well-resolved simulation data. On the other hand, it is important to note that the knowledge drawn from moderate $R e$ simulations is insightful for the prediction at high $R e$, since the variation of mean flow property with $R e$ is governed by symmetries. This is the essence of the SED theory, though it needs to be tested by further studies. Note also that the SED approach rejects the proposal of a universal mathematical model for turbulent mean flows (such as $k-\epsilon$ or $k-\omega$ or any of its variants), which are currently the basis for industrial fluid dynamics modeling and which seem to become increasingly complicated, rendering CFD increasingly more artistic with compromising accuracy. The SED approach proposes a new line of thinking: using DNS information to derive simple and physically sound CFD models. The latter will be a research target in the near future.

In addition to developing an analysis platform for guiding DNS studies, we are also interested in deriving a rational theory of turbulence, for such canonical flows as channel, pipe, or boundary layer. We believe that, after an extended SED analysis of DNS data, mechanisms that connect the fluctuations to the mean would be quantitatively characterized, and then a consistent model and theory using physically sound assumptions (starting from EANS equations), may arise. Such a theory would explain the behavior of the order functions in DNS data, and make predictions for other $R e$. Examination of the models for several flows would allow us to identify universal components in the theory. Only then, a viable theory will emerge. We end this paper with a statement reflecting a century's fight behind turbulence research: Law is not universal, but the way of searching is!

Acknowledgments. Special thanks go to X.L. Li who supply us with his compressible TBL simulation code and some data, and Y.S. Zhang for analyzing TBL data. The authors have benefitted from discussions with a number of colleagues, including S.Y. Chen, C.B. Lee, J. Chen, W.T. Bi, J.J. Tao, Y.P. Shi, and students N. Hu, J. Pei, Y.Z. Wang, Z.X. Zhang, J.H. Xie.

Open Access This article is distributed under the terms of the Creative Commons Attribution Noncommercial License which permits any noncommercial use, distribution, and reproduction in any medium, provided the original author(s) and source are credited.

\section{References}

1. Kolmogorov, A.N.: Equations of turbulent motion of an incompressible viscous fluid. Izv. Acad. Sci. USSR Phys. 6(1-2), 56-58 (1942)

2. Robinson, S.K.: Coherent motions in the turbulent boundary layer. Annu. Rev. Fluid Mech. 23, 601-639 (1991)

3. Moin, P., Mahesh, K.: Direct numerical simulation: A tool in turbulence research. Annu. Rev. Fluid Mech. 30, 539-578 (1998) 
4. Schoppa, W., Hussain, F.: Coherent structure generation in nearwall turbulence. J. Fluid Mech. 453, 57-108 (2002)

5. Prandtl, L.: Zur turbulenten Strömung in Rohren und laengs Platten. Ergeb. Aerodyn. Versuch Geottingen IV. Lieferung 4, 18-29 (1932)

6. Karman, T.: Mechanische Ahnlichkeit und Turbulenz. Nachr Ges Wiss Gottingen, Math Phys Klasse 58C76. 2 (1930)

7. Clauser, F.H.: The turbulent boundary layer. Adv. Appl. Mech. 4, 2C51.2 (1956)

8. George, W.K., Castillo, L.: Zero-pressure-gradient turbulent boundary layer. Appl. Mech. Rev. 50, 689-729 (1997)

9. Jones, M.B., Nickels, T.B., Marusic, I.: On the asymptotic similarity of the zero-pressure-gradient turbulent boundary layer. J. Fluid. Mech. 616, 195-203 (2008)

10. Zagarola, M.V., Smits, A.J.: Scaling of the mean velocity profile for tuebulent pipe flow. Phys. Rev. Lett. 78, 2 (1997)

11. George, W.K.: Recent advancements toward the understanding of turbulent boundary layers. In: Proceedings of the Fourth AIAA Theoretical Fluid Mechanics Meeting, Toronto, Canada, 6-9 June 2005, AIAA Paper 2005-4669

12. Millikan, C.B.: A critical discussion of turbulent flows in channelsand circular tubes. In: Den Hartog, J.P., Peters, H. (eds.) Proceedings of the Fifth International Congress on Applied Mechanics, pp. 386-392. Wiley, New York (1938)

13. Nickels, T.B.: Inner scaling for wall-bounded flows subject to large pressure gradients. J. Fluid Mech. 521, 217-239 (2004)

14. Monkewitz, P.A., Chauhan, K.A., Nagib, H.M.: Self-consistent high-Reynolds-number asymptotics for zero-pressure-gradient turbulent boundary layers. Phys. Fluids 19, 115101-115112 (2007)

15. Wosnik, M., Castillo, L., Geroge, W.K.: A theory for turbulent pipe and channel flows. J. Fluid Mech. 421, 115-145 (2000)

16. Buschmanna, M.H., Gad-el-Hak, M.: Recent developments in scaling of wall-bounded flows. Prog. Aerosp. Sci. 42, 419-467 (2007)

17. Barenblatt, G.I.: Scaling laws for fully developed turbulent shear flows. Part 1,Basic hypotheses and analysis. J. Fluid Mech. 248, 513-520 (1993)

18. Barenblatt, G.I., Chorin, A.J., Prostokishin, V.M.: Self-similar intermediate structures in turbulent boundary layers at large Reynolds numbers. J. Fluid Mech. 410, 263-283 (2000)

19. Sreenivansan, R.: The importance of higher-order effects in the Barenblatt-Chorin theory of wall-bounded fully developed turbulent shear flows. Phys. Fluids 10, 1037C9 (1998)

20. Oberlack, M.: Symmetries, invariance and scaling-Laws in inhomogeneous turbulent shear flows. Flow Turbulence Combus. 62, 111-135 (1999)

21. Oberlack, M.: A unified approach for symmetries in plane parallel turbulent shear flows. Flow Turbulence Combus. 427, 299-328 (2001)
22. L'vov, V.S., Procaccia, I., Rudenco, O.: Universal model of finite Reynolds number turbulent flow in channels and pipes. Phys. Rev. Let. 100(1-4), 050504 (2008)

23. Zagarola, M.V., Perry, A.E., Smits, A.J.: Laws or power laws: The scaling in the overlap region. Phys. Fluids 9, 2094 (1997)

24. George, W.K.: Is there a universal log-law for turbulent wallbounded flow? Phil. Trans. Roy. Soc. A 365, 789-806 (2007)

25. Nagib, H.M., Chauhan, K.A.: Variations of von Kármán coefficient in canonical flows. Phys. Fluids 20, 101518 (2008)

26. Monkewitz, P.A., Chauhan, K.A., Nagib, H.M.: of mean flow similarity laws in zero pressure gradient turbulent boundary layers. Phys. Fluids 20, 105102 (2008)

27. Oberlack, M., Rosteck, A.: New statistical symmetries of the multi-point equations and its importance for turbulent scaling laws. Discret. Contin. Dyn. Syst. Ser. S 3(3), 451-471 (2010)

28. Marusic, I., McKeon, B.J., Monkewitz, P.A., et al.: Comparison of mean flow similarity laws in zero pressure gradient turbulent boundary layers. Phys. Fluids 22, 065103 (2010)

29. She, Z.S., Leveque, E.: Universal scaling laws in fully developed turbulence. Phys. Rev. Lett. 72, 336 (1994)

30. She, Z.S., Zhang, Z.X.: Universal hierarchical symmetry for turbulence and general multi-scale fluctuation systems. Acta Mech. Sin. 25, 279-294 (2009)

31. Hoyas, S., Jimenez, J.: Scaling of the velocity fluctuations in turbulent channels up to $R e_{\tau}=2003$. Phys. Fluids 18, 011702 (2006). http://torroja.dmt.upm.es/ftp/channels/

32. Gao, H., Fu, D.-X., Ma, Y.-W., et al.: Direct numerical simulation of supersonic turbulent boundary layer flow. Chin. Phys. Lett. 22, 1709 (2005)

33. Iwamoto, K., Suzuki, Y., Kasagi, N.: Database of fully developed channel flow. THTLAB Internal Report No. ILR-0201 (2002). http://www.thtlab.t.u-tokyo.ac.jp

34. Batchelor, G.K.: Pressure fluctuations in isotropic turbulence. In: Proceedings of Cambridge Philosophical Society 47, 359 (1951)

35. Pope, S.B.: Turbulent Flows. Cambridge Univ. Press, Cambridge (2000)

36. She, Z.S., Hu, N., Wu, Y.: Structural ensemble dynamics based closure model for wall-bounded turbulent flow. Acta. Mech. Sin. 25, 731-736 (2009)

37. Bradshaw, P., Ferriss, D.H., Atwell, N.P.: Calculation of boundary layer development using the turbulent energy equation. J. Fluid Mech. 28, 593-616 (1967)

38. Wilcox, D.C.: Turbulence Modeling for CFD. DCW Industries, Philadelphia (2006)

39. Moser, R.D., Kim, J., Mansour, N.N.: Direct numerical simulation of turbulent channel flow up to $R e=590$. Phys. Fluids 11, 943945 (1999) 